\title{
Enhanced Symplectic Synchronization between Two Different Complex Chaotic Systems with Uncertain Parameters
}

\author{
Cheng-Hsiung Yang \\ Graduate Institute of Automation and Control, National Taiwan University of Science and Technology, 43 Section 4, \\ Keelung Road, Taipei 106, Taiwan
}

Correspondence should be addressed to Cheng-Hsiung Yang; chyang123123@mail.ntust.edu.tw

Received 12 October 2012; Accepted 13 April 2013

Academic Editor: Haydar Akca

Copyright (C) 2013 Cheng-Hsiung Yang. This is an open access article distributed under the Creative Commons Attribution License, which permits unrestricted use, distribution, and reproduction in any medium, provided the original work is properly cited.

An enhanced symplectic synchronization of complex chaotic systems with uncertain parameters is studied. The traditional chaos synchronizations are special cases of the enhanced symplectic synchronization. A sufficient condition is given for the asymptotical stability of the null solution of error dynamics. The enhanced symplectic synchronization may be applied to the design of secure communication. Finally, numerical simulations results are performed to verify and illustrate the analytical results.

\section{Introduction}

A synchronized mechanism that enables a system to maintain a desired dynamical behavior (the goal or target) even when intrinsically chaotic has many applications ranging from biology to engineering [1-4]. Thus, it is of considerable interest and potential utility to devise control techniques capable of achieving the desired type of behavior in nonlinear and chaotic systems. Many approaches have been presented for the synchronization of chaotic systems [5-10]. There are a chaotic master system and either an identical or a different slave system. Our goal is the synchronization of the chaotic master and the chaotic slave by coupling or by other methods.

The symplectic chaos synchronization concept [11]

$$
y=H(t, x, y)+F(t)
$$

is studied, where $x, y$ are the state vectors of the master system and of the slave system, respectively, and $F(t)$ is a given function of time in different form. The $F(t)$ may be a regular motion function or a chaotic motion function. When $H(t, x, y)+F(t)=x$ and $H(t, x, y)=x$, (1) reduces to the generalized chaos synchronization and the traditional chaos synchronization given in [1-3], respectively. In this paper, a new enhance symplectic chaos synchronization:

$$
y=H(t, \dot{x}, \dot{y}, x, y)+F(t) .
$$

As numerical examples, we select hyperchaotic Chen system [12] and hyperchaotic Lorenz system [13] as the master system and the slave system, respectively.

This paper is organized as follows. In Section 2, by the Lyapunov asymptotical stability theorem, a symplectic synchronization scheme is given. In Section 3, various feedbacks of nonlinear controllers are designed for the enhanced symplectic synchronization of a hyperchaotic Chen system with uncertain parameters and a hyperchaotic Lorenz system. Numerical simulations are also given in Section 3. Finally, some concluding remarks are given in Section 4.

\section{Enhanced Symplectic Synchronization Scheme}

There are two different nonlinear chaotic systems. The partner $A$ controls the partner $B$ partially. The partner $A$ is given by

$$
\dot{x}=f(t, x, A(t)),
$$

where $x=\left[x_{1}, x_{2}, \ldots, x_{n}\right]^{T} \in R^{n}$ is a state vector, $A(t)=$ $\left[A_{1}(t), A_{2}(t), \ldots, A_{M}(t)\right]^{T} \in R^{M}$ is a vector of uncertain coefficients in $f$, and $f$ is a vector function. 
The partner $B$ is given by

$$
\dot{y}=g(t, y, B(t)) \text {, }
$$

where $y=\left[y_{1}, y_{2}, \ldots, y_{n}\right]^{T} \in R^{n}$ is a state vector, $B(t)=$ $\left[B_{1}(t), B_{2}(t), \ldots, B_{m}(t)\right]^{T} \in R^{m}$ is a vector of uncertain coefficients in $g$, and $g$ is a vector function different from $f$.

After a controller $u(t)$ is added, partner $B$ becomes

$$
\dot{y}=g(t, y, B(t))+u(t),
$$

where $u(t)=\left[u_{1}(t), u_{2}(t), \ldots, u_{n}(t)\right]^{T} \in R^{n}$ is the control vector.

Our goal is to design the controller $u(t)$ so that the state vector $y$ of the partner $B$ asymptotically approaches $H(t, \dot{x}, \dot{y}, x, y)+F(t)$, a given function $H(t, \dot{x}, \dot{y}, x, y)$ plus a given vector function $F(t)=\left[F_{1}(t), F_{2}(t), \ldots, F_{n}(t)\right]^{T}$ which is a regular or a chaotic function. Define error vector $e(t)=$ $\left[e_{1}, e_{2}, \ldots, e_{n}\right]^{T}$ :

$$
\begin{gathered}
e=H(t, \dot{x}, \dot{y}, x, y)-y+F(t), \\
\lim _{t \rightarrow \infty} e=0
\end{gathered}
$$

is demanded.

From (5), it is obtained that

$$
\dot{e}=\frac{\partial H}{\partial t}+\nabla H^{T} \dot{\Psi}-\dot{y}+\dot{F}(t)
$$

where $\dot{\Psi}=\left[\begin{array}{llll}\ddot{x} & \ddot{y} & \dot{x} & \dot{y}\end{array}\right]^{T}$.

Using (3), (4a), and (4b), (7) can be rewritten as

$$
\begin{aligned}
\dot{e}= & \frac{\partial H}{\partial t}+\frac{\partial H}{\partial \dot{x}} \ddot{x}+\frac{\partial H}{\partial \dot{y}} \ddot{y}+\frac{\partial H}{\partial x} f(t, x, A(t)) \\
& +\frac{\partial H}{\partial y} g(t, y, B(t))-g(t, y, B(t))-u(t)+\dot{F}(t) .
\end{aligned}
$$

Proof. A positive definite Lyapunov function $V(e)$ is chosen $[14,15]$ as

$$
V(e)=\frac{1}{2} e^{T} e .
$$

Its derivative along any solution of (8) is

$$
\begin{aligned}
\dot{V}(e)=e^{T}\{ & \frac{\partial H}{\partial t}+\frac{\partial H}{\partial \dot{x}} \ddot{x}+\frac{\partial H}{\partial \dot{y}} \ddot{y}+\frac{\partial H}{\partial x} \\
& \times f(t, x, A(t))+\frac{\partial H}{\partial y} g(t, y, B(t)) \\
& -g(t, y, B(t))+\dot{F}(t)-u(t)\} .
\end{aligned}
$$

In (10), the $u(t)$ is designed so that $\dot{V}(e)=e^{T} C_{n \times n} e$, where $C_{n \times n}$ is a diagonal negative definite matrix. The $\dot{V}$ is a negative definite function of $e$.

Remark 1. Note that e approaches zero when time approaches infinitly, according to Lyapunov theorem of asymptotical stability. The enhanced symplectic synchronization is obtained $[12,13,16-19]$.

\section{Numerical Results for the Enhanced Symplectic Chaos Synchronization of Chen System with Uncertain Parameters and Hyperchaotic Lorenz System}

To further illustrate the effectiveness of the controller, we select hyperchaotic Chen system and hyperchaotic Lorenz system as the master system and the slave system, respectively. Consider

$$
\begin{aligned}
& \dot{x}_{1}=a\left(x_{2}-x_{1}\right)+x_{4}, \\
& \dot{x}_{2}=d x_{1}+c x_{2}-x_{1} x_{3}, \\
& \dot{x}_{3}=-b x_{3}+x_{1} x_{2}, \\
& \dot{x}_{4}=r x_{4}+x_{2} x_{3}, \\
& \dot{y}_{1}=a_{1}\left(y_{2}-y_{1}\right)+y_{4}, \\
& \dot{y}_{2}=b_{1} y_{1}-y_{2}-y_{1} y_{3}, \\
& \dot{y}_{3}=-c_{1} y_{3}+y_{1} y_{2}, \\
& \dot{y}_{4}=d_{1} y_{4}-y_{1} y_{3},
\end{aligned}
$$

where $a, b, c, d, r, a_{1}, b_{1}, c_{1}$, and $d_{1}$ are parameters. The parameters of master system and slave system are chosen as $a=31, b=3.5, c=11, d=7.7, r=0.1, a_{1}=11, b_{1}=$ 28, $c_{1}=2.8$, and $d_{1}=1.2$.

The controllers $u_{1}, u_{2}, u_{3}$, and $u_{4}$ are added to the four equations of (12), respectively as follows:

$$
\begin{aligned}
& \dot{y}_{1}=a_{1}\left(y_{2}-y_{1}\right)+y_{4}+u_{1}, \\
& \dot{y}_{2}=b_{1} y_{1}-y_{2}-y_{1} y_{3}+u_{2}, \\
& \dot{y}_{3}=-c_{1} y_{3}+y_{1} y_{2}+u_{3}, \\
& \dot{y}_{4}=d_{1} y_{4}-y_{1} y_{3}+u_{4} .
\end{aligned}
$$

The initial values of the states of the Chen system and of the Lorenz system are taken as $x_{1}(0)=11, x_{2}(0)=13, x_{3}(0)$ $=12, x_{4}(0)=12, y_{1}(0)=-11, y_{2}(0)=-13, y_{3}(0)=-12$, and $y_{4}(0)=-12$.

Case 1 (a symplectic synchronization). We take $F_{1}(t)=x_{4}^{3}(t)$, $F_{2}(t)=x_{1}^{3}(t), F_{3}(t)=x_{2}^{3}(t)$, and $F_{4}(t)=x_{3}^{3}(t)$. They are chaotic functions of time. $H_{i}(x, y, t)=-x_{i}^{2} y_{i}(i=1,2,3,4)$ are given. By (6), we have

$$
\begin{array}{r}
\lim _{t \rightarrow \infty} e_{i}=\lim _{t \rightarrow \infty}\left(-x_{i}^{2} y_{i}-y_{i}+x_{j}^{3}\right)=0, \\
i=1,2,3,4 ; j= \begin{cases}4, & i=1, \\
i-1, & i \neq 1 .\end{cases}
\end{array}
$$

From (7), we have

$$
\begin{array}{r}
\dot{e}_{i}=-2 \dot{x}_{i} x_{i} y_{i}-x_{i}^{2} \dot{y}_{i}-\dot{y}_{i}+3 \dot{x}_{j} x_{j}^{2}, \\
i=1,2,3,4 ; j= \begin{cases}4, & i=1, \\
i-1, & i \neq 1 .\end{cases}
\end{array}
$$


Equation (8) can be expressed as

$$
\begin{aligned}
\dot{e}_{1}= & -2 y_{1} x_{1}\left[a\left(x_{2}-x_{1}\right)+x_{4}\right] \\
& -\left(1+x_{1}^{2}\right) \times\left[a_{1}\left(y_{2}-y_{1}\right)+y_{4}\right] \\
& -u_{1}+3 x_{4}^{2}\left(r x_{4}+x_{2} x_{3}\right), \\
\dot{e}_{2}= & -2 y_{2} x_{2}\left(d x_{1}+c x_{2}-x_{1} x_{3}\right) \\
& -\left(1+x_{2}^{2}\right) \times\left(b_{1} y_{1}-y_{2}-y_{1} y_{3}\right) \\
& -u_{2}+3 x_{1}^{2}\left[a\left(x_{2}-x_{1}\right)+x_{4}\right], \\
\dot{e}_{3}= & -2 y_{3} x_{3}\left(-b x_{3}+x_{1} x_{2}\right) \\
& -\left(1+x_{3}^{2}\right) \times\left(-c_{1} y_{3}+y_{1} y_{2}\right) \\
& -u_{3}+3 x_{2}^{2}\left(d x_{1}+c x_{2}-x_{1} x_{3}\right), \\
\dot{e}_{4}= & -2 y_{4} x_{4}\left(r x_{4}+x_{2} x_{3}\right) \\
& -\left(1+x_{4}^{2}\right) \times\left(d_{1} y_{4}-y_{1} y_{3}\right) \\
& -u_{4}+3 x_{3}^{2}\left(-b x_{3}+x_{1} x_{2}\right),
\end{aligned}
$$

where $e_{1}=-x_{1}^{2} y_{1}-y_{1}+x_{4}^{3}, e_{2}=-x_{2}^{2} y_{2}-y_{2}+x_{1}^{3}, e_{3}=$ $-x_{3}^{2} y_{3}-y_{3}+x_{2}^{3}$, and $e_{4}=-x_{4}^{2} y_{4}-y_{4}+x_{3}^{3}$.

Choose a positive definite Lyapunov function as

$$
V\left(e_{1}, e_{2}, e_{3}, e_{4}\right)=\frac{1}{2}\left(e_{1}^{2}+e_{2}^{2}+e_{3}^{2}+e_{4}^{2}\right) .
$$

Its time derivative along any solution of (16) is

$$
\begin{aligned}
& \dot{V}=e_{1}\{-2 y_{1} x_{1}\left[a\left(x_{2}-x_{1}\right)+x_{4}\right] \\
&-\left(1+x_{1}^{2}\right) \times\left[a_{1}\left(y_{2}-y_{1}\right)+y_{4}\right]-u_{1} \\
&+\left.3 x_{4}^{2}\left(r x_{4}+x_{2} x_{3}\right)\right\} \\
&+e_{2}\{-2 y_{2} x_{2}\left(d x_{1}+c x_{2}-x_{1} x_{3}\right) \\
&-\left(1+x_{2}^{2}\right) \times\left(b_{1} y_{1}-y_{2}-y_{1} y_{3}\right) \\
&\left.-u_{2}+3 x_{1}^{2}\left[a\left(x_{2}-x_{1}\right)+x_{4}\right]\right\} \\
&+e_{3}\left\{-2 y_{3} x_{3}\left(-b x_{3}+x_{1} x_{2}\right)\right. \\
&-\left(1+x_{3}^{2}\right) \times\left(-c_{1} y_{3}+y_{1} y_{2}\right)-u_{3} \\
&\left.+3 x_{2}^{2}\left(d x_{1}+c x_{2}-x_{1} x_{3}\right)\right\} \\
&+e_{4}\left\{-2 y_{4} x_{4}\left(r x_{4}+x_{2} x_{3}\right)\right. \\
&-\left(1+x_{4}^{2}\right) \times\left(d_{1} y_{4}-y_{1} y_{3}\right) \\
&\left.-u_{4}+3 x_{3}^{2}\left(-b x_{3}+x_{1} x_{2}\right)\right\}
\end{aligned}
$$

According to (10), we get the controller

$$
\begin{aligned}
u_{1}= & -2 y_{1} x_{1}\left[a\left(x_{2}-x_{1}\right)+x_{4}\right]+3 x_{4}^{2}\left(r x_{4}+x_{2} x_{3}\right) \\
& -\left(1+x_{1}^{2}\right) \times\left[a_{1}\left(y_{2}-y_{1}\right)+y_{4}\right]+e_{1}, \\
u_{2}= & -2 y_{2} x_{2}\left(d x_{1}+c x_{2}-x_{1} x_{3}\right)+3 x_{1}^{2}\left[a\left(x_{2}-x_{1}\right)+x_{4}\right] \\
& -\left(1+x_{2}^{2}\right) \times\left(b_{1} y_{1}-y_{2}-y_{1} y_{3}\right)+e_{2}, \\
u_{3}= & -2 y_{3} x_{3}\left(-b x_{3}+x_{1} x_{2}\right)+3 x_{2}^{2}\left(d x_{1}+c x_{2}-x_{1} x_{3}\right) \\
& +\left(1+x_{3}^{2}\right) \times\left(c_{1} y_{3}-y_{1} y_{2}\right)+e_{3}, \\
u_{4}= & -2 y_{4} x_{4}\left(r x_{4}+x_{2} x_{3}\right)+3 x_{3}^{2}\left(-b x_{3}+x_{1} x_{2}\right) \\
& -\left(1+x_{4}^{2}\right) \times\left(d_{1} y_{4}-y_{1} y_{3}\right)+e_{4} .
\end{aligned}
$$

Equation (18) becomes

$$
\dot{V}=-\left(e_{1}^{2}+e_{2}^{2}+e_{3}^{2}+e_{4}^{2}\right)<0,
$$

which is negative definite. The Lyapunov asymptotical stability theorem is satisfied. The symplectic synchronization of the Chen system and the Lorenz system is achieved. The numerical results are shown in Figures 1, 2, and 3. After 1 second, the motion trajectories enter a chaotic attractor.

Case 2 (a symplectic synchronization with uncertain parameters). The master Chen system with uncertain variable parameters is

$$
\begin{aligned}
& \dot{x}_{1}=a(t)\left(x_{2}-x_{1}\right)+x_{4}, \\
& \dot{x}_{2}=d(t) x_{1}+c(t) x_{2}-x_{1} x_{3}, \\
& \dot{x}_{3}=-b(t) x_{3}+x_{1} x_{2}, \\
& \dot{x}_{4}=r(t) x_{4}+x_{2} x_{3},
\end{aligned}
$$

where $a(t), b(t), c(t), d(t)$, and $r(t)$ are uncertain parameters. In simulation, we take

$$
\begin{gathered}
a(t)=a\left(1+k_{1} \sin \omega_{1} t\right), \quad b(t)=b\left(1+k_{2} \sin \omega_{2} t\right), \\
c(t)=c\left(1+k_{3} \sin \omega_{3} t\right), \quad d(t)=d\left(1+k_{4} \sin \omega_{4} t\right), \\
r(t)=r\left(1+k_{5} \sin \omega_{5} t\right),
\end{gathered}
$$

where $k_{1}, k_{2}, k_{3}, k_{4}, k_{5}, \omega_{1}, \omega_{2}, \omega_{3}, \omega_{4}$, and $\omega_{5}$ are constants. Take $k_{1}=0.3, k_{2}=0.5, k_{3}=0.2, k_{4}=0.4, k_{5}=0.6, \omega_{1}=$ $13, \omega_{2}=17, \omega_{3}=19, \omega_{4}=23$, and $\omega_{5}=29$. So, (21) is chaotic system, shown in Figure 4 .

We take $F_{1}(t)=x_{4}^{3}(t), F_{2}(t)=x_{1}^{3}(t), F_{3}(t)=x_{2}^{3}(t)$, and $F_{4}(t)=x_{3}^{3}(t)$. They are chaotic functions of time. $H_{i}(x, y, t)=$ $-x_{i}^{2} y_{i}(i=1,2,3,4)$ are given. By $(6)$, we have

$$
\begin{array}{r}
\lim _{t \rightarrow \infty} e_{i}=\lim _{t \rightarrow \infty}\left(-x_{i}^{2} y_{i}-y_{i}+x_{j}^{3}\right)=0, \\
i=1,2,3,4 ; j= \begin{cases}4, & i=1, \\
i-1, & i \neq 1 .\end{cases}
\end{array}
$$




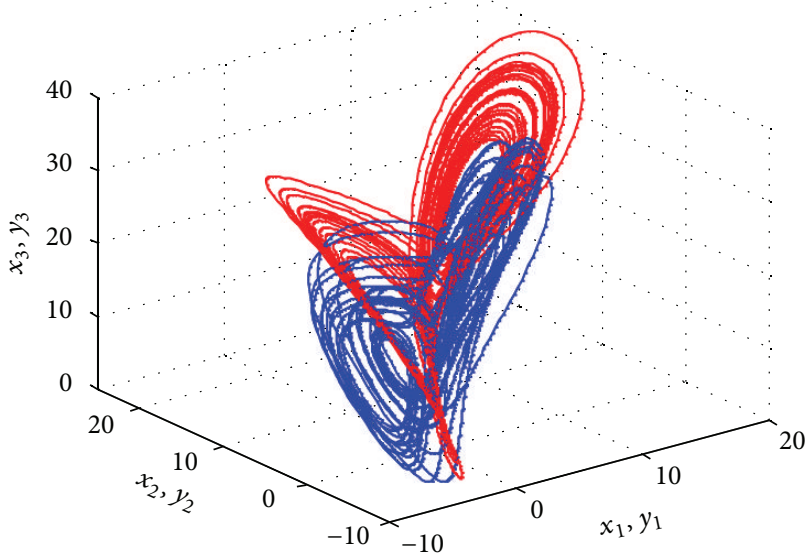

(a)

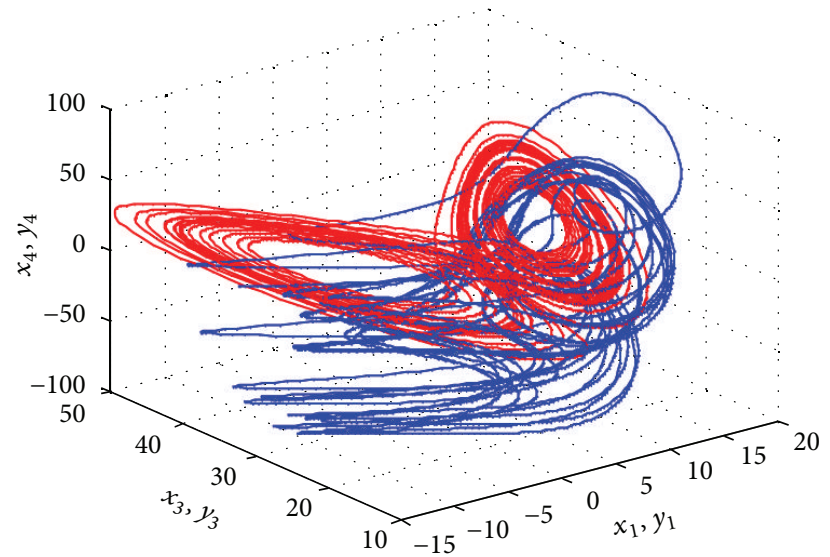

— Master system

— Slave system

(c)

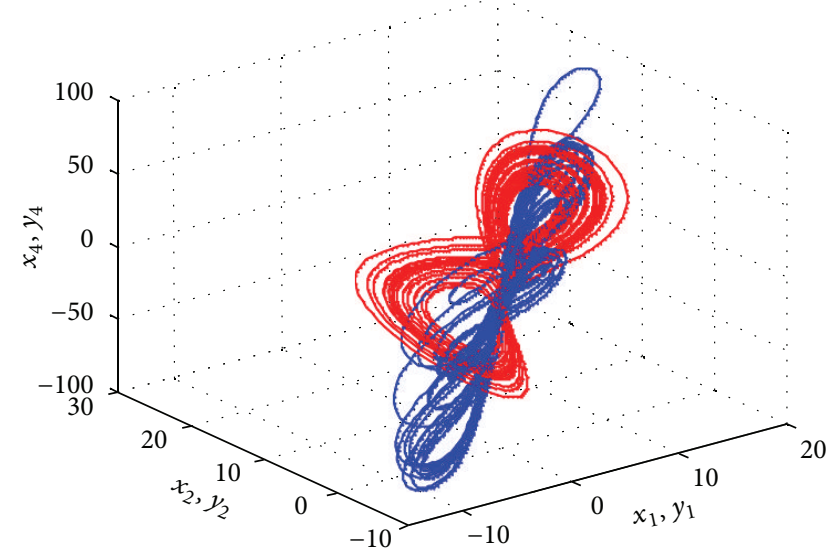

(b)

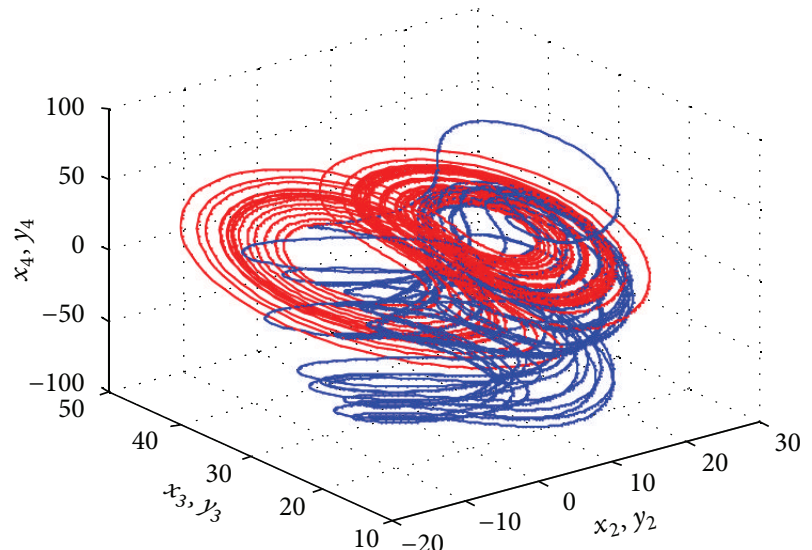

_ Master system

(d)

FIGURE 1: Projections of phase portrait for master system (11) and slave system (12).

From (7), we have

$$
\begin{array}{r}
\dot{e}_{i}=-2 \dot{x}_{i} x_{i} y_{i}-x_{i}^{2} \dot{y}_{i}-\dot{y}_{i}+3 \dot{x}_{j} x_{j}^{2}, \\
i=1,2,3,4 ; j= \begin{cases}4, & i=1, \\
i-1, & i \neq 1 .\end{cases}
\end{array}
$$

Equation (8) can be expressed as

$$
\begin{aligned}
\dot{e}_{1}= & -2 y_{1} x_{1}\left[a(t)\left(x_{2}-x_{1}\right)+x_{4}\right] \\
& -\left(1+x_{1}^{2}\right) \times\left[a_{1}\left(y_{2}-y_{1}\right)+y_{4}+u_{1}\right] \\
& +3 x_{4}^{2}\left(r(t) x_{4}+x_{2} x_{3}\right), \\
\dot{e}_{2}= & -2 y_{2} x_{2}\left(d(t) x_{1}+c(t) x_{2}-x_{1} x_{3}\right) \\
& -\left(1+x_{2}^{2}\right) \times\left(b_{1} y_{1}-y_{2}-y_{1} y_{3}+u_{2}\right) \\
& +3 x_{1}^{2}\left[a(t)\left(x_{2}-x_{1}\right)+x_{4}\right],
\end{aligned}
$$

$$
\begin{aligned}
\dot{e}_{3}= & -2 y_{3} x_{3}\left(-b(t) x_{3}+x_{1} x_{2}\right) \\
& -\left(1+x_{3}^{2}\right) \times\left(-c_{1} y_{3}+y_{1} y_{2}+u_{3}\right) \\
& +3 x_{2}^{2}\left(d(t) x_{1}+c(t) x_{2}-x_{1} x_{3}\right), \\
\dot{e}_{4}= & -2 y_{4} x_{4}\left(r(t) x_{4}+x_{2} x_{3}\right) \\
& -\left(1+x_{4}^{2}\right) \times\left(d_{1} y_{4}-y_{1} y_{3}+u_{4}\right) \\
& +3 x_{3}^{2}\left(-b(t) x_{3}+x_{1} x_{2}\right),
\end{aligned}
$$

where $e_{1}=-x_{1}^{2} y_{1}-y_{1}+x_{4}^{3}, e_{2}=-x_{2}^{2} y_{2}-y_{2}+x_{1}^{3}, e_{3}=$ $-x_{3}^{2} y_{3}-y_{3}+x_{2}^{3}$, and $e_{4}=-x_{4}^{2} y_{4}-y_{4}+x_{3}^{3}$.

Choose a positive definite Lyapunov function as

$$
V\left(e_{1}, e_{2}, e_{3}, e_{4}\right)=\frac{1}{2}\left(e_{1}^{2}+e_{2}^{2}+e_{3}^{2}+e_{4}^{2}\right) .
$$




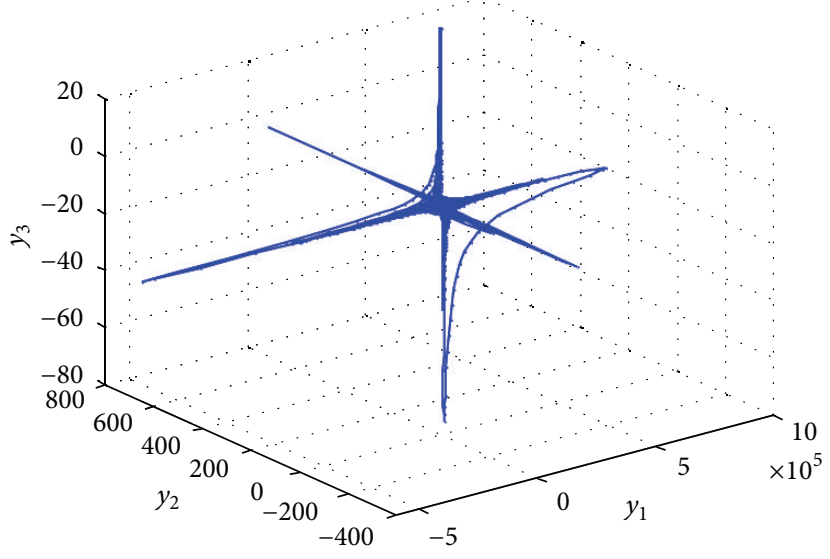

(a)

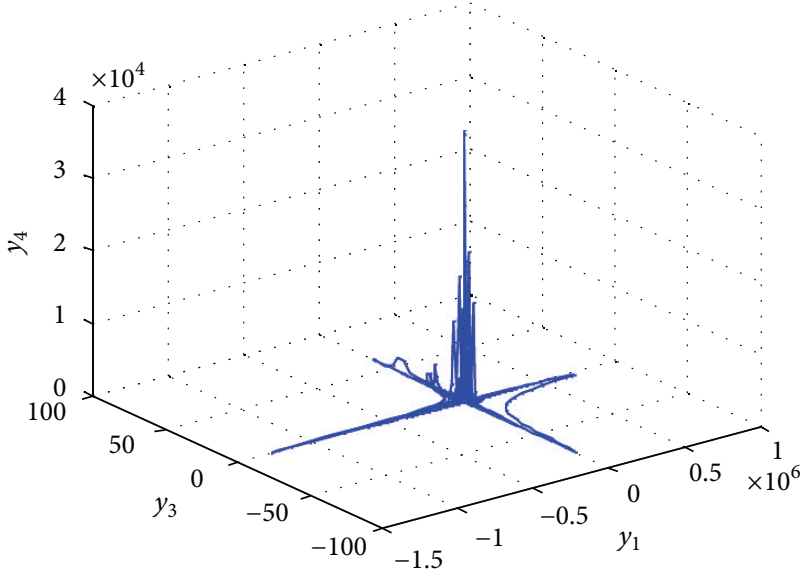

(c)

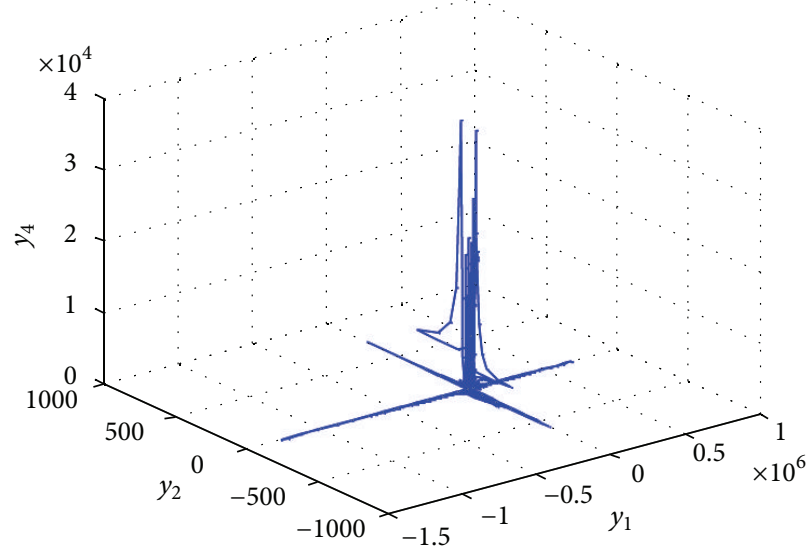

(b)

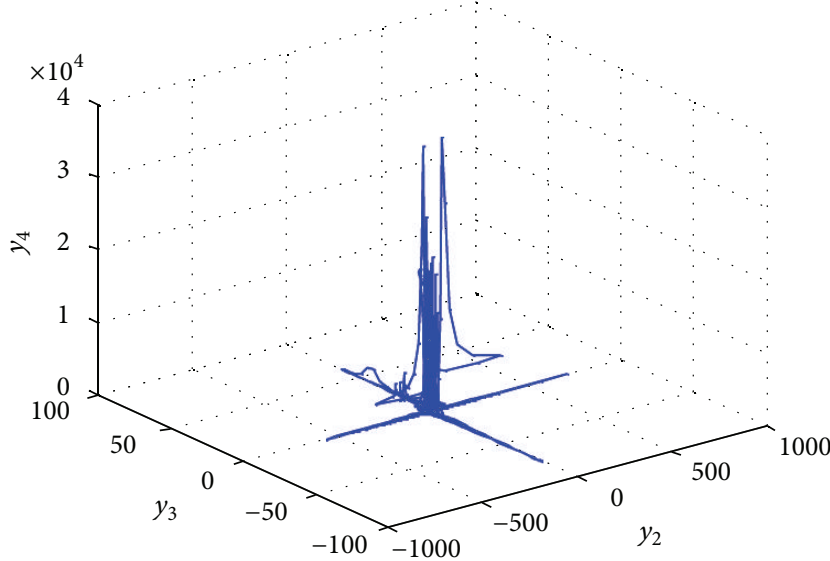

(d)

Figure 2: Projections of the phase portrait for chaotic system (13) of Case 1.

Its time derivative along any solution of (25) is

$$
\begin{aligned}
& \dot{V}=e_{1}\{-2 y_{1} x_{1}\left[a(t)\left(x_{2}-x_{1}\right)+x_{4}\right] \\
&-\left(1+x_{1}^{2}\right) \times\left[a_{1}\left(y_{2}-y_{1}\right)+y_{4}\right]-u_{1} \\
&+\left.3 x_{4}^{2}\left(r(t) x_{4}+x_{2} x_{3}\right)\right\} \\
&+e_{2}\left\{-2 y_{2} x_{2}\left(d(t) x_{1}+c(t) x_{2}-x_{1} x_{3}\right)\right. \\
&-\left(1+x_{2}^{2}\right) \times\left(b_{1} y_{1}-y_{2}-y_{1} y_{3}\right)-u_{2} \\
&\left.+3 x_{1}^{2}\left[a(t)\left(x_{2}-x_{1}\right)+x_{4}\right]\right\} \\
&+e_{3}\left\{-2 y_{3} x_{3}\left(-b(t) x_{3}+x_{1} x_{2}\right)\right. \\
&-\left(1+x_{3}^{2}\right) \times\left(-c_{1} y_{3}+y_{1} y_{2}\right)-u_{3} \\
&\left.+3 x_{2}^{2}\left(d(t) x_{1}+c(t) x_{2}-x_{1} x_{3}\right)\right\} \\
&+e_{4}\left\{-2 y_{4} x_{4}\left(r(t) x_{4}+x_{2} x_{3}\right)\right. \\
&-\left(1+x_{4}^{2}\right) \times\left(d_{1} y_{4}-y_{1} y_{3}\right) \\
&\left.-u_{4}+3 x_{3}^{2}\left(-b(t) x_{3}+x_{1} x_{2}\right)\right\} .
\end{aligned}
$$

According to (10), we get the controller

$$
\begin{aligned}
u_{1}= & -2 y_{1} x_{1}\left[a(t)\left(x_{2}-x_{1}\right)+x_{4}\right] \\
& +3 x_{4}^{2}\left(r(t) x_{4}+x_{2} x_{3}\right) \\
& -\left(1+x_{1}^{2}\right)\left[a_{1}\left(y_{2}-y_{1}\right)-y_{4}\right]+e_{1}, \\
u_{2}= & -2 y_{2} x_{2}\left(d(t) x_{1}+c(t) x_{2}-x_{1} x_{3}\right) \\
& +3 x_{1}^{2}\left[a(t)\left(x_{2}-x_{1}\right)+x_{4}\right] \\
& -\left(1+x_{2}^{2}\right)\left(b_{1} y_{1}-y_{2}-y_{1} y_{3}\right)+e_{2}, \\
u_{3}= & -2 y_{3} x_{3}\left(-b(t) x_{3}+x_{1} x_{2}\right) \\
& +3 x_{2}^{2}\left(d(t) x_{1}+c(t) x_{2}-x_{1} x_{3}\right) \\
& +\left(1+x_{3}^{2}\right)\left(c_{1} y_{3}-y_{1} y_{2}\right)+e_{3}, \\
u_{4}= & -2 y_{4} x_{4}\left(r(t) x_{4}+x_{2} x_{3}\right) \\
& +3 x_{3}^{2}\left(-b(t) x_{3}+x_{1} x_{2}\right) \\
& -\left(1+x_{4}^{2}\right)\left(d_{1} y_{4}-y_{1} y_{3}\right)+e_{4} .
\end{aligned}
$$




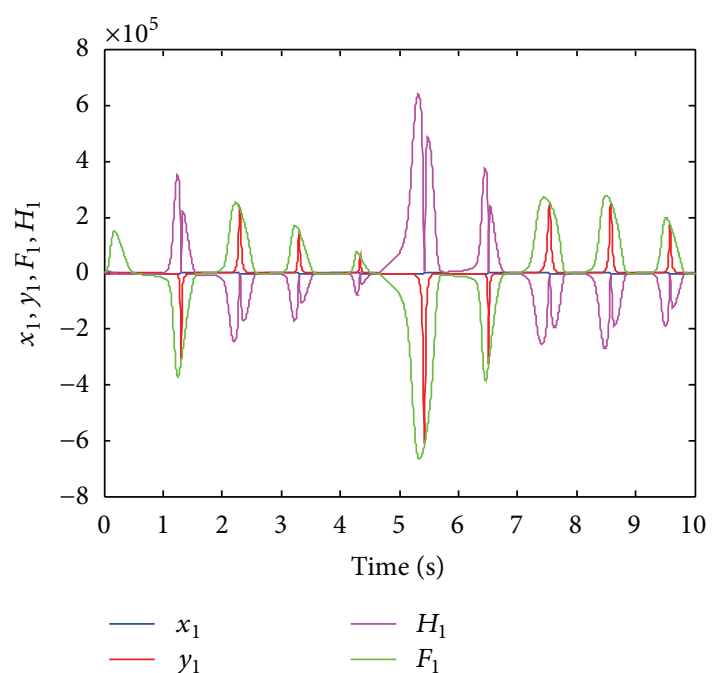

(a)
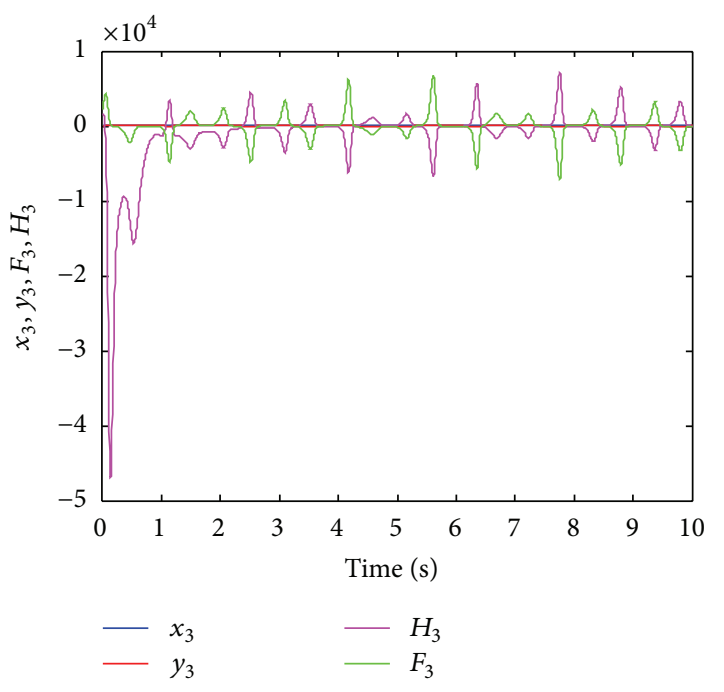

(c)

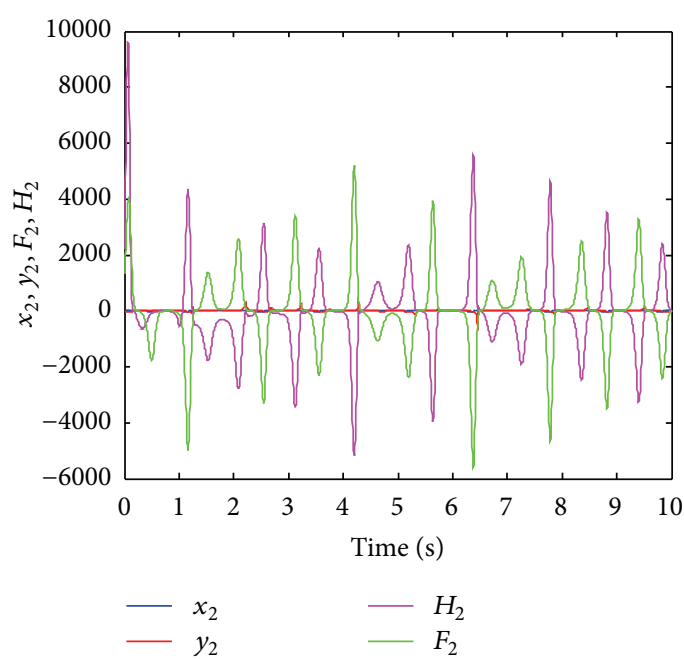

(b)

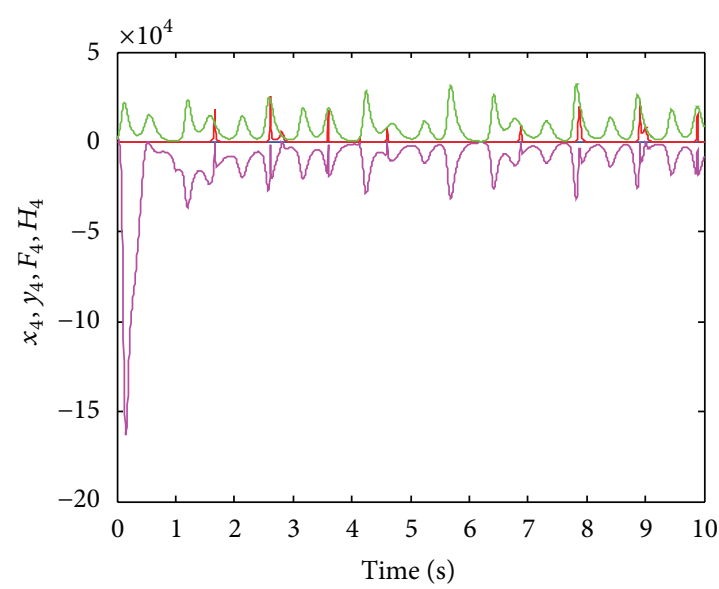

(d)

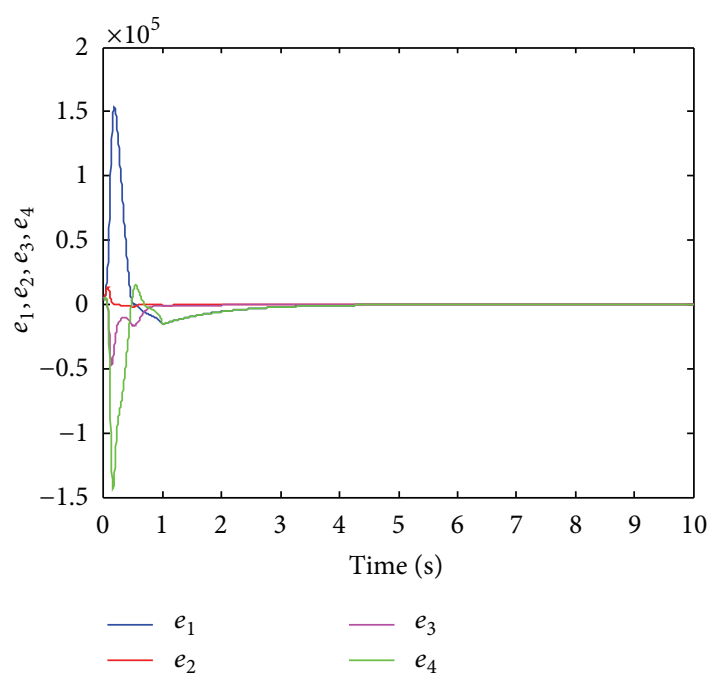

(e)

Figure 3: Time histories of states, state errors, $F_{1}, F_{2}, F_{3}, F_{4}, H_{1}, H_{2}, H_{3}$, and $H_{4}$ for Case 1. 


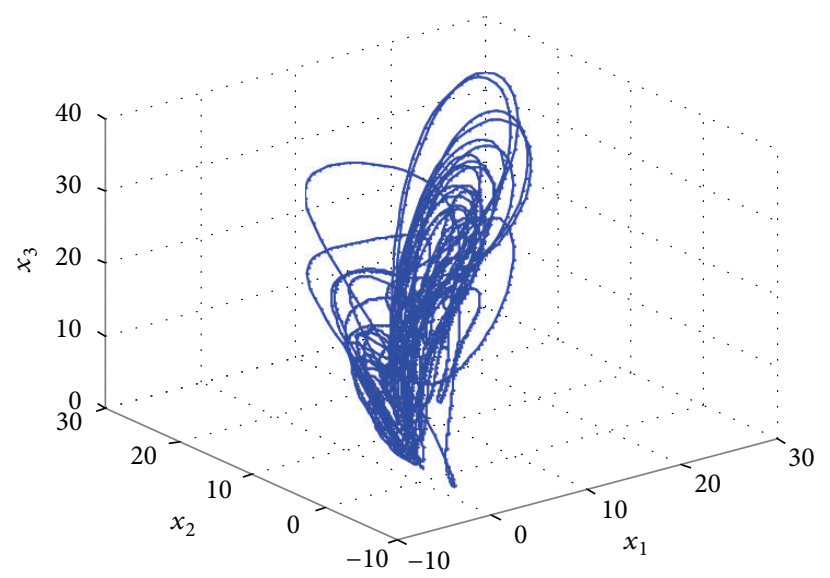

(a)

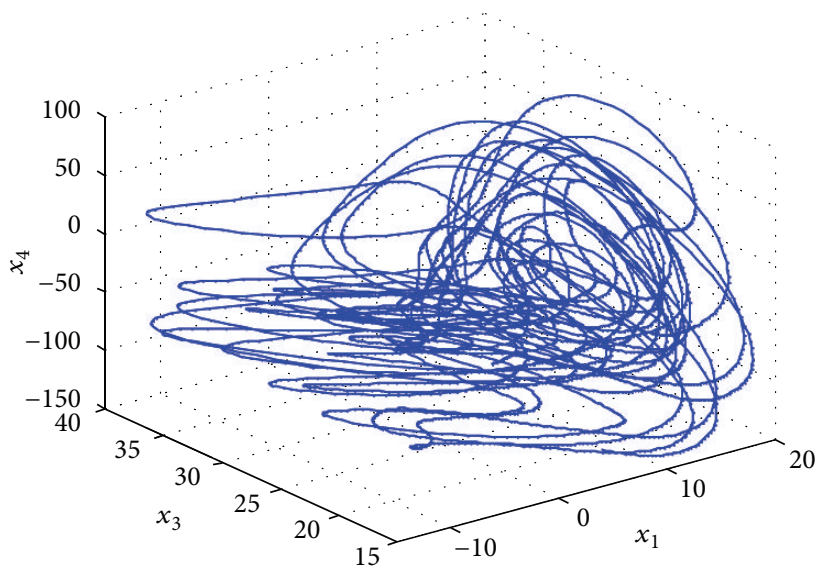

(c)

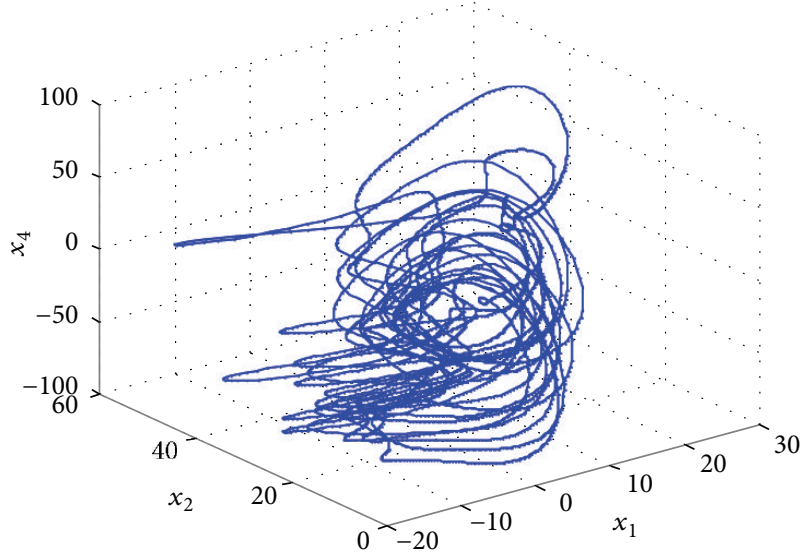

(b)

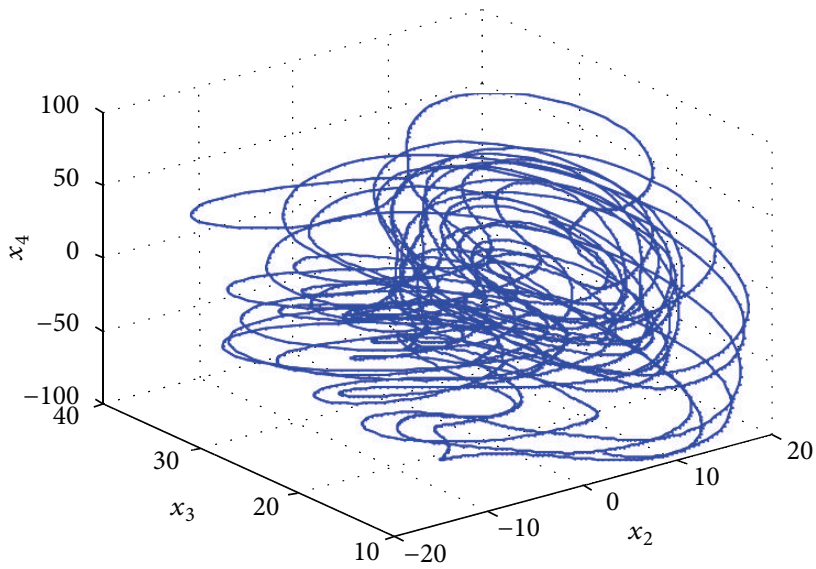

(d)

FIGURE 4: Projections of the phase portrait for chaotic system (21).

Equation (27) becomes

$$
\dot{V}=-\left(e_{1}^{2}+e_{2}^{2}+e_{3}^{2}+e_{4}^{2}\right)<0
$$

which is negative definite. The Lyapunov asymptotical stability theorem is satisfied. The symplectic synchronization of the Chen system with uncertain parameters and the Lorenz system is achieved. The numerical results are shown in Figures 5 and 6 . After 1 second, the motion trajectories enter a chaotic attractor.

Case 3 (an enhanced symplectic synchronization with uncertain parameters). We take $F_{1}(t)=x_{4}^{3}(t), F_{2}(t)=$ $x_{1}^{3}(t), F_{3}(t)=x_{2}^{3}(t)$, and $F_{4}(t)=x_{3}^{3}(t)$. They are chaotic functions of time. $H_{i}(\dot{x}, \dot{y}, x, y, t)=-x_{i}^{2} y_{i}-\dot{x}-K \dot{y}(i=$ $1,2,3,4)$ are given. The $K$ value is 0.0001 . By (6), we have

$$
\begin{array}{r}
\lim _{t \rightarrow \infty} e_{i}=\lim _{t \rightarrow \infty}\left(-x_{i}^{2} y_{i}-\dot{x}_{i}-K \dot{y}_{i}-y_{i}+x_{j}^{3}\right)=0, \\
i=1,2,3,4 ; j= \begin{cases}4, & i=1, \\
i-1, & i \neq 1 .\end{cases}
\end{array}
$$

From (7) we have

$$
\begin{array}{r}
\dot{e}_{i}=-2 x_{i} \dot{x}_{i} y_{i}-x_{i}^{2} \dot{y}_{i}-\ddot{x}_{i}-K \ddot{y}_{i}-\dot{y}_{i}+3 \dot{x}_{j} x_{j}^{2}, \\
i=1,2,3,4 ; j= \begin{cases}4, & i=1, \\
i-1, & i \neq 1 .\end{cases}
\end{array}
$$

Equation (8) can be expressed as

$$
\begin{aligned}
\dot{e}_{1}= & -2 y_{1} x_{1}\left[a(t)\left(x_{2}-x_{1}\right)+x_{4}\right] \\
& -\left(1+x_{1}^{2}\right) \times\left[a_{1}\left(y_{2}-y_{1}\right)+y_{4}+u_{1}\right] \\
& -\ddot{x}_{1}-K \ddot{y}_{1}+3 x_{4}^{2}\left(r(t) x_{4}+x_{2} x_{3}\right), \\
\dot{e}_{2}= & -2 y_{2} x_{2}\left(d(t) x_{1}+c(t) x_{2}-x_{1} x_{3}\right) \\
& -\left(1+x_{2}^{2}\right) \times\left(b_{1} y_{1}-y_{2}-y_{1} y_{3}+u_{2}\right) \\
& -\ddot{x}_{2}-K \ddot{y}_{2}+3 x_{1}^{2}\left[a(t)\left(x_{2}-x_{1}\right)+x_{4}\right], \\
\dot{e}_{3}= & -2 y_{3} x_{3}\left(-b(t) x_{3}+x_{1} x_{2}\right) \\
& -\left(1+x_{3}^{2}\right) \times\left(-c_{1} y_{3}+y_{1} y_{2}+u_{3}\right)
\end{aligned}
$$




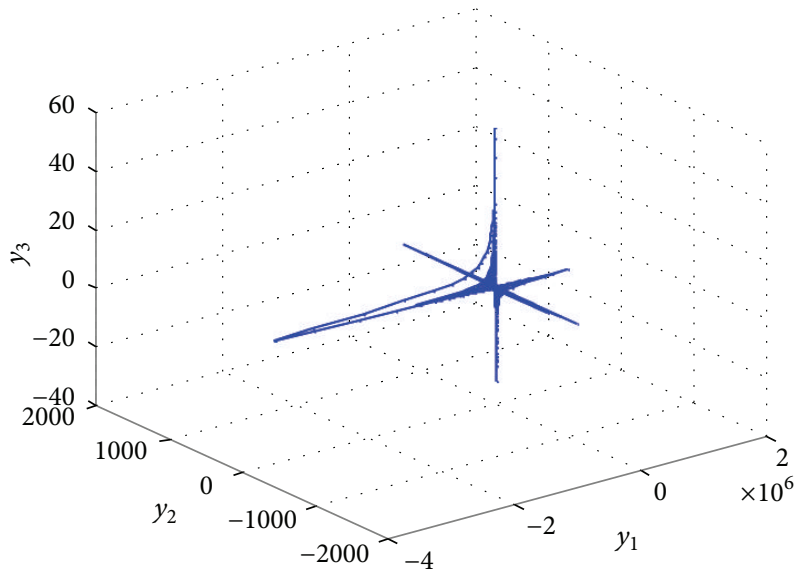

(a)

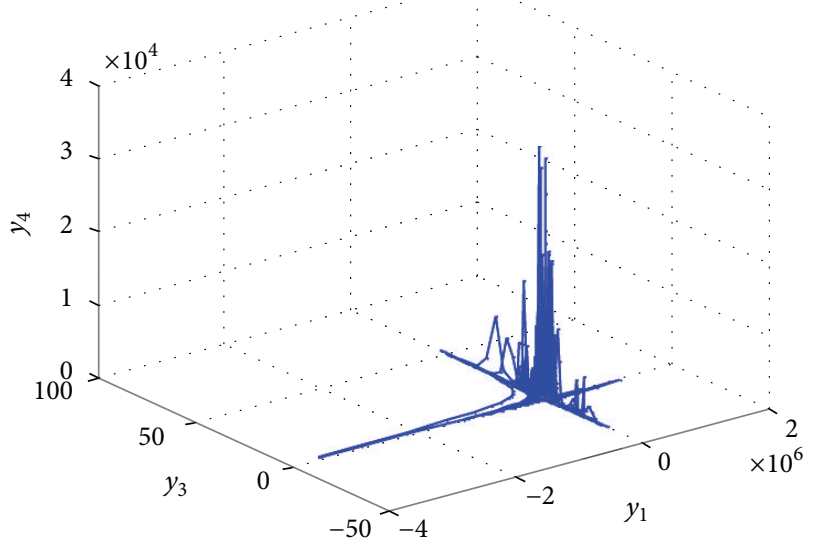

(c)

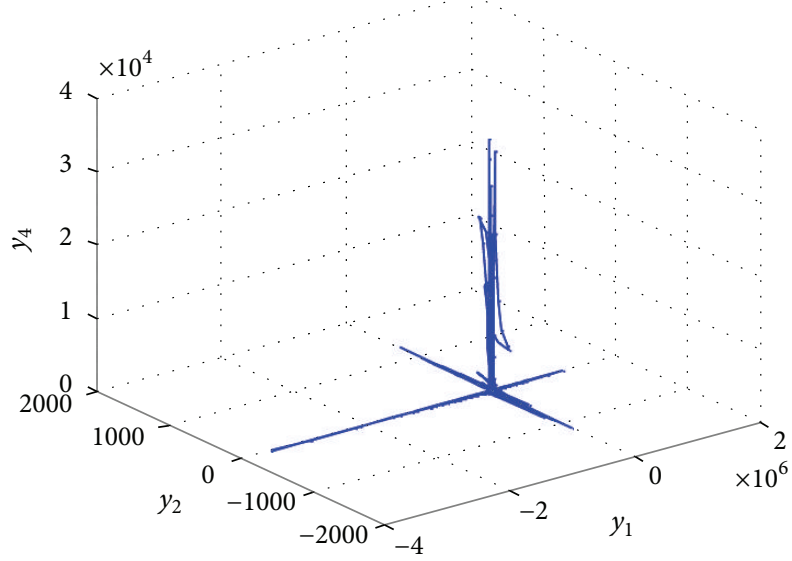

(b)

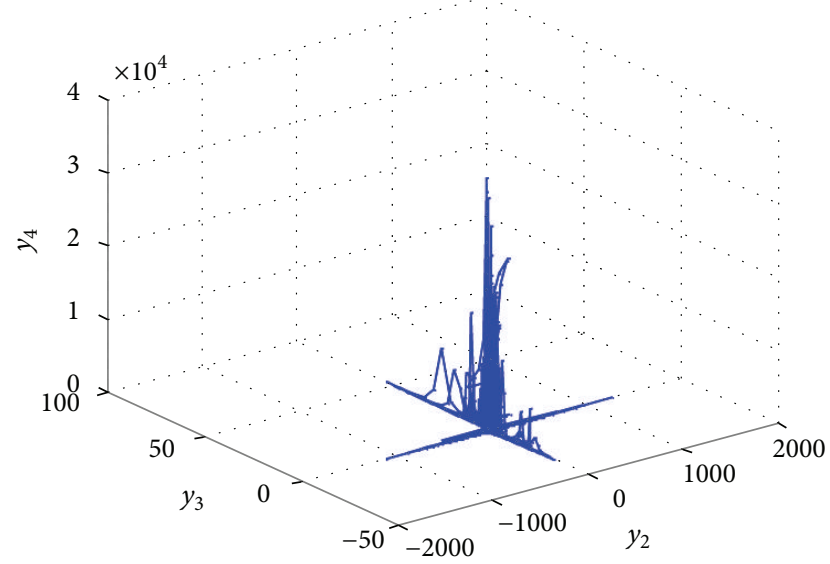

(d)

FIGURE 5: Projections of the phase portrait for chaotic system (13) of Case 2.

$$
\begin{aligned}
& -\ddot{x}_{3}-K \ddot{y}_{3}+3 x_{2}^{2}\left(d(t) x_{1}+c(t) x_{2}-x_{1} x_{3}\right), \\
\dot{e}_{4}= & -2 y_{4} x_{4}\left(r(t) x_{4}+x_{2} x_{3}\right) \\
& -\left(1+x_{4}^{2}\right) \times\left(d_{1} y_{4}-y_{1} y_{3}+u_{4}\right) \\
& -\ddot{x}_{4}-K \ddot{y}_{4}+3 x_{3}^{2}\left(-b(t) x_{3}+x_{1} x_{2}\right),
\end{aligned}
$$

where $e_{1}=-x_{1}^{2} y_{1}-y_{1}-\dot{x}_{1}-K \dot{y}_{1}+x_{4}^{3}, e_{2}=-x_{2}^{2} y_{2}-y_{2}-$ $\dot{x}_{2}-K \dot{y}_{2}+x_{1}^{3}, e_{3}=-x_{3}^{2} y_{3}-y_{3}-\dot{x}_{3}-K \dot{y}_{3}+x_{2}^{3}$, and $e_{4}=$ $-x_{4}^{2} y_{4}-y_{4}-\dot{x}_{4}-K \dot{y}_{4}+x_{3}^{3}$.

Choose a positive definite Lyapunov function as

$$
V\left(e_{1}, e_{2}, e_{3}, e_{4}\right)=\frac{1}{2}\left(e_{1}^{2}+e_{2}^{2}+e_{3}^{2}+e_{4}^{2}\right)
$$

Its time derivative along any solution of (32) is

$$
\begin{aligned}
\dot{V}=e_{1}\{ & -2 y_{1} x_{1}\left[a(t)\left(x_{2}-x_{1}\right)+x_{4}\right] \\
& -\left(1+x_{1}^{2}\right) \times\left[a_{1}\left(y_{2}-y_{1}\right)+y_{4}\right]+u_{1}
\end{aligned}
$$

$$
\begin{aligned}
- & \left.\ddot{x}_{1}-K \ddot{y}_{1}+3 x_{4}^{2}\left(r(t) x_{4}+x_{2} x_{3}\right)\right\} \\
+e_{2}\{ & -2 y_{2} x_{2}\left(d(t) x_{1}+c(t) x_{2}-x_{1} x_{3}\right) \\
& -\left(1+x_{2}^{2}\right) \times\left(b_{1} y_{1}-y_{2}-y_{1} y_{3}\right)+u_{2} \\
& \left.-\ddot{x}_{2}-K \ddot{y}_{2}+3 x_{1}^{2}\left[a(t)\left(x_{2}-x_{1}\right)+x_{4}\right]\right\} \\
+e_{3}\{ & -2 y_{3} x_{3}\left(-b(t) x_{3}+x_{1} x_{2}\right) \\
& -\left(1+x_{3}^{2}\right) \times\left(-c_{1} y_{3}+y_{1} y_{2}\right)+u_{3} \\
& \left.-\ddot{x}_{3}-K \ddot{y}_{3}+3 x_{2}^{2}\left(d(t) x_{1}+c(t) x_{2}-x_{1} x_{3}\right)\right\}
\end{aligned}
$$$$
+e_{4}\left\{-2 y_{4} x_{4}\left(r(t) x_{4}+x_{2} x_{3}\right)\right.
$$$$
-\left(1+x_{4}^{2}\right) \times\left(d_{1} y_{4}-y_{1} y_{3}\right)+u_{4}
$$$$
\left.-\ddot{x}_{4}-K \ddot{y}_{4}+3 x_{3}^{2}\left(-b(t) x_{3}+x_{1} x_{2}\right)\right\} .
$$ 

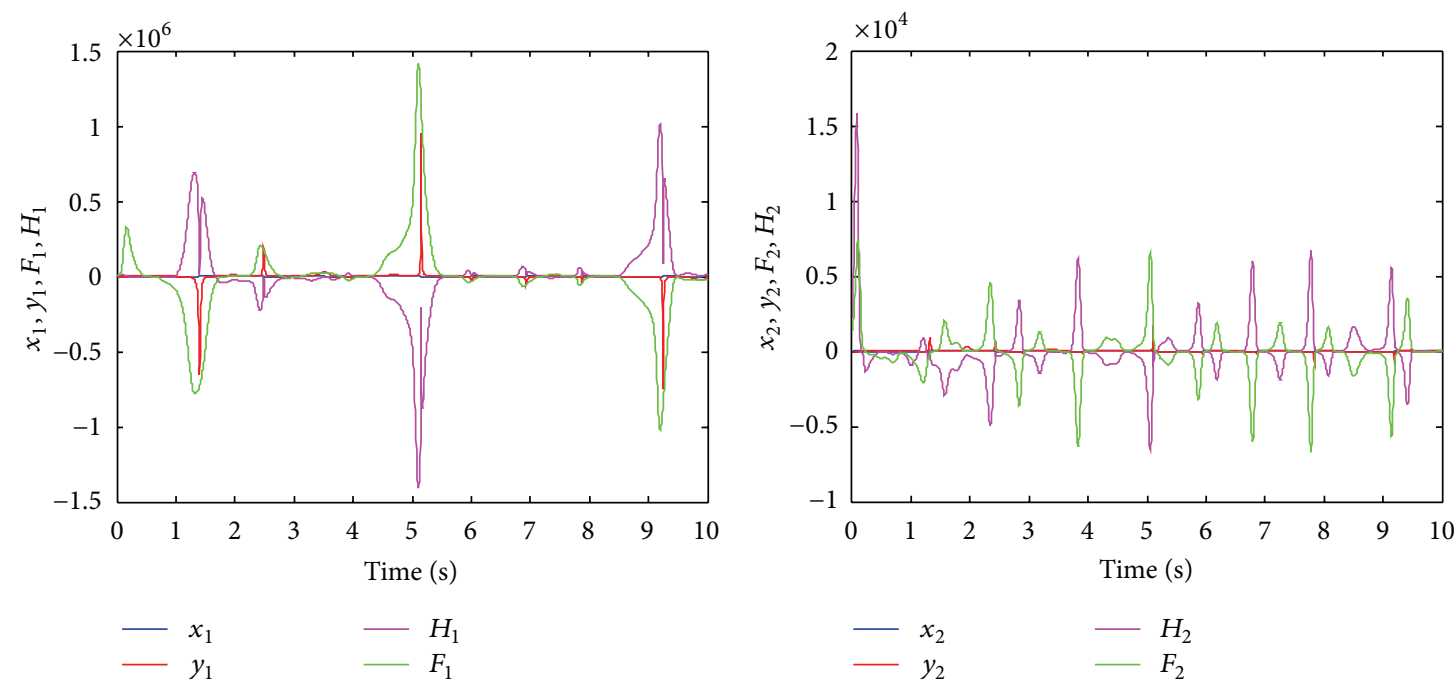

(a)

(b)
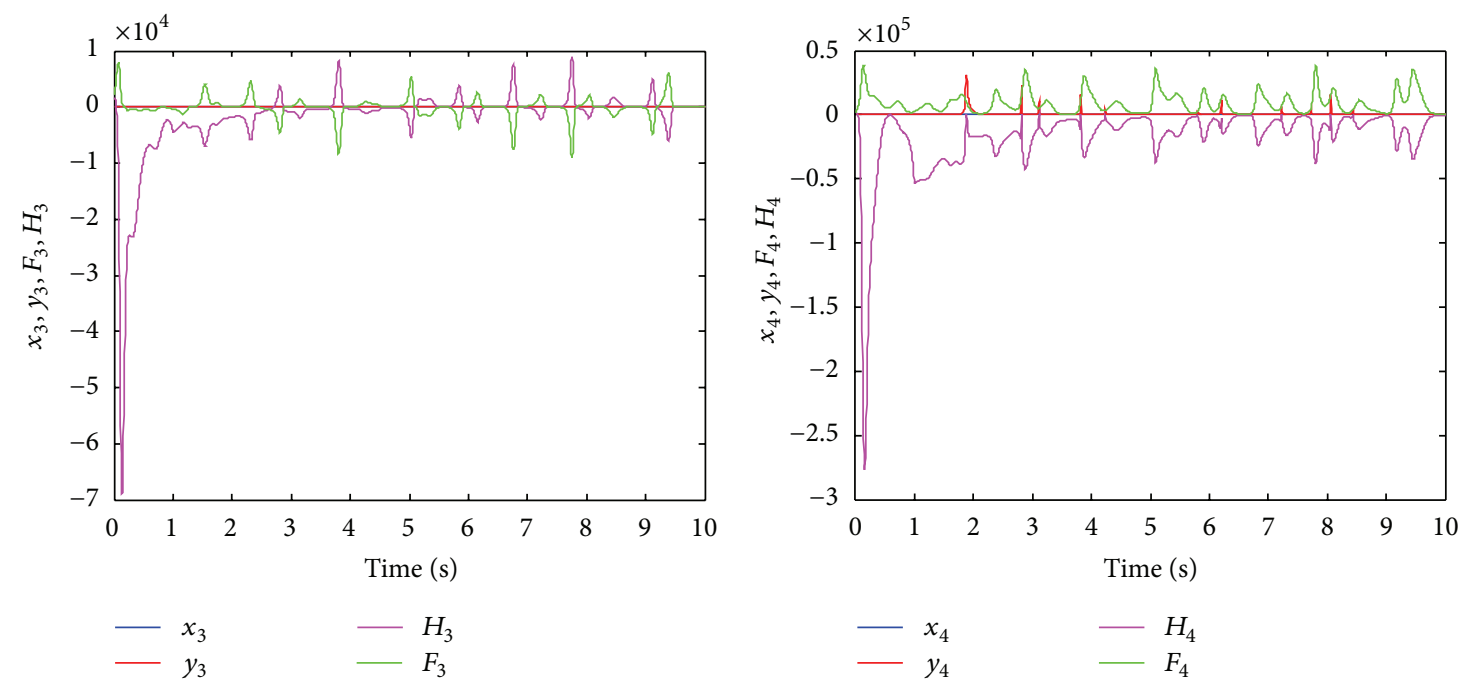

(c)

(d)

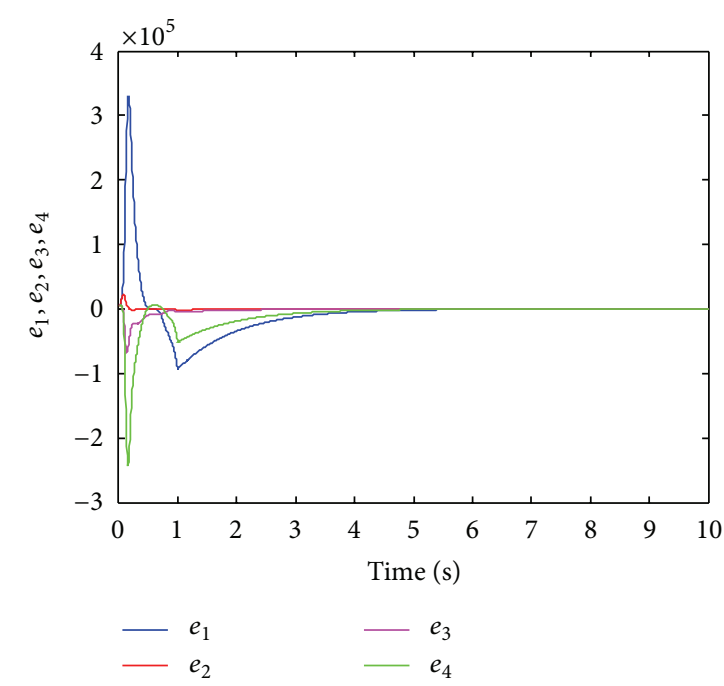

(e)

Figure 6: Time histories of states, state errors, $F_{1}, F_{2}, F_{3}, F_{4}, H_{1}, H_{2}, H_{3}$, and $H_{4}$ for Case 2. 


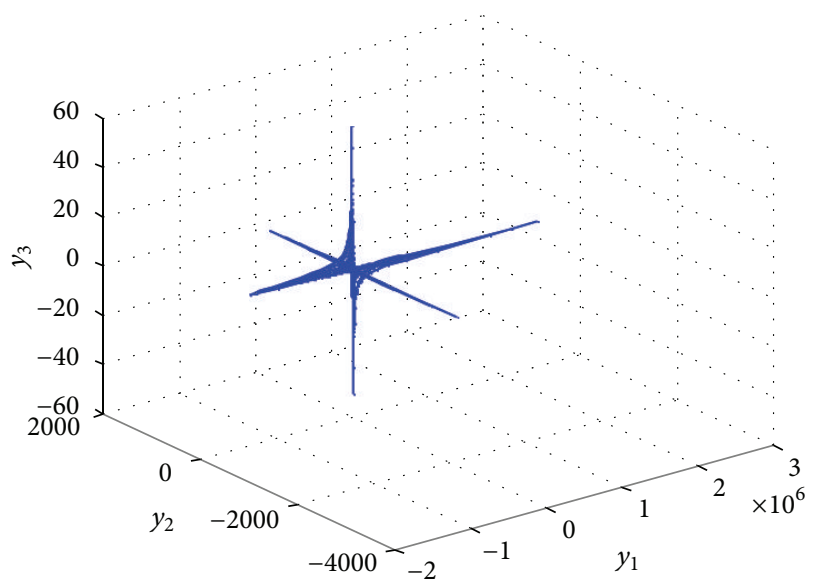

(a)

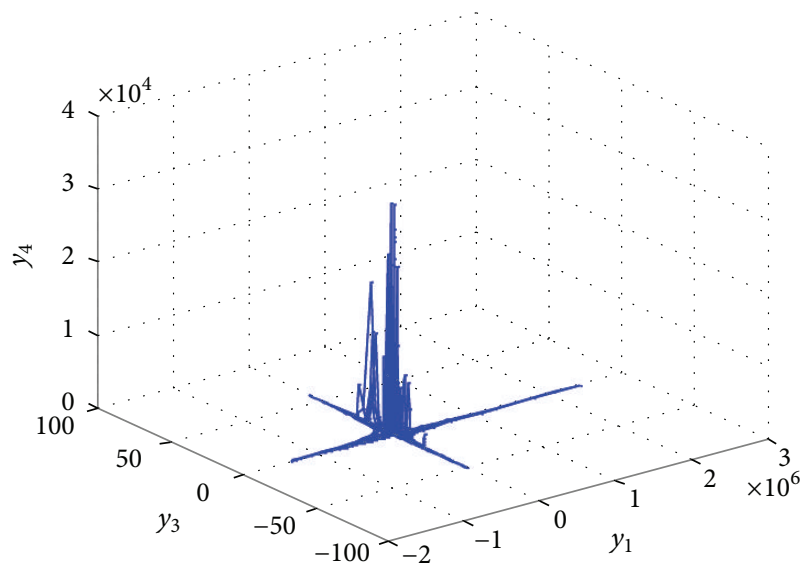

(c)

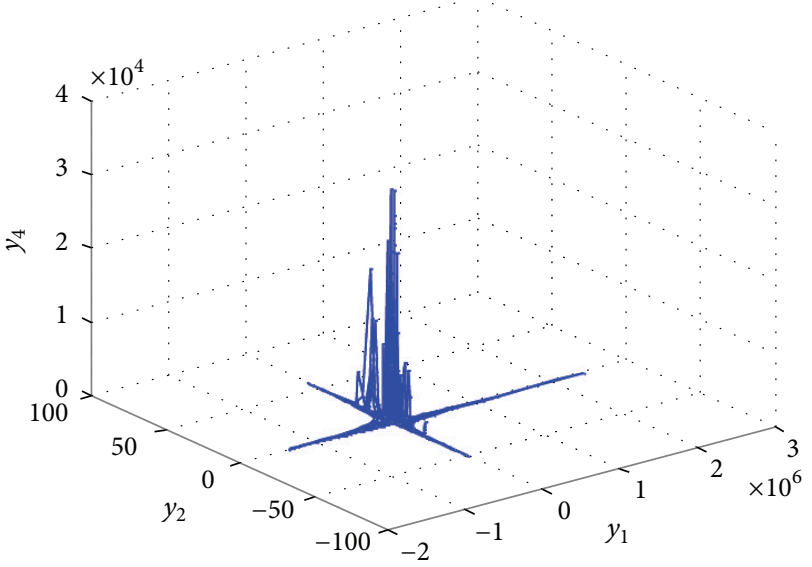

(b)

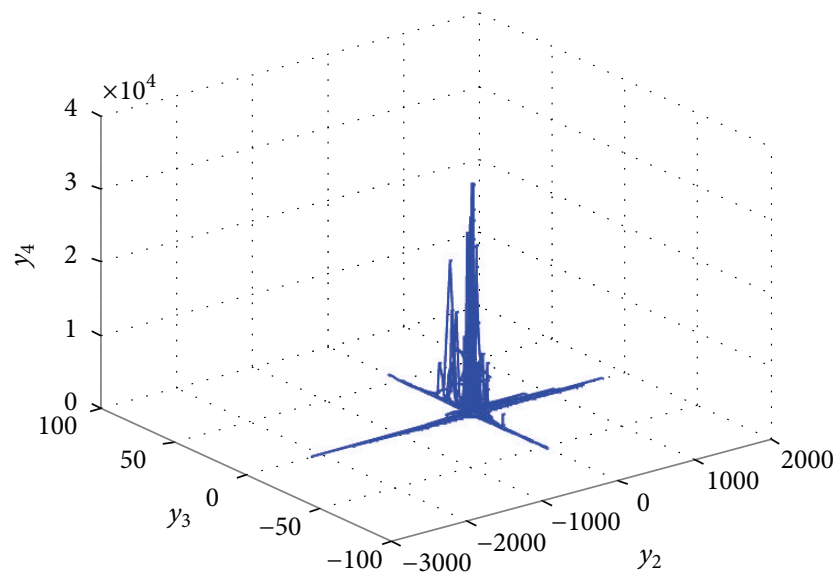

(d)

Figure 7: Projections of the phase portrait for chaotic system (13) of Case 3.

According to (10), we get the controller

$$
\begin{aligned}
u_{1}= & -2 y_{1} x_{1}\left[a(t)\left(x_{2}-x_{1}\right)+x_{4}\right] \\
& -\ddot{x}_{1}-K \ddot{y}_{1}+3 x_{4}^{2}\left(r(t) x_{4}+x_{2} x_{3}\right) \\
& -\left(1+x_{1}^{2}\right)\left[a_{1}\left(y_{2}-y_{1}\right)+y_{4}\right]+e_{1}, \\
u_{2}= & -2 y_{2} x_{2}\left(d(t) x_{1}+c(t) x_{2}-x_{1} x_{3}\right) \\
& -\ddot{x}_{2}-K \ddot{y}_{2}+3 x_{1}^{2}\left[a(t)\left(x_{2}-x_{1}\right)+x_{4}\right] \\
& -\left(1+x_{2}^{2}\right)\left(b_{1} y_{1}-y_{2}-y_{1} y_{3}\right)+e_{2}, \\
u_{3}= & -2 y_{3} x_{3}\left(-b(t) x_{3}+x_{1} x_{2}\right) \\
& -\ddot{x}_{3}-K \ddot{y}_{3}+3 x_{2}^{2}\left(d(t) x_{1}+c(t) x_{2}-x_{1} x_{3}\right) \\
& +\left(1+x_{3}^{2}\right)\left(c_{1} y_{3}-y_{1} y_{2}\right)+e_{3}, \\
u_{4}= & -2 y_{4} x_{4}\left(r(t) x_{4}+x_{2} x_{3}\right)-\ddot{x}_{4}
\end{aligned}
$$

$$
\begin{aligned}
& -K \ddot{y}_{4}+3 x_{3}^{2}\left(-b(t) x_{3}+x_{1} x_{2}\right) \\
& -\left(1+x_{4}^{2}\right)\left(d_{1} y_{4}-y_{1} y_{3}\right)+e_{4} .
\end{aligned}
$$

Equation (34) becomes

$$
\dot{V}=-\left(e_{1}^{2}+e_{2}^{2}+e_{3}^{2}+e_{4}^{2}\right)<0,
$$

which is negative definite. The Lyapunov asymptotical stability theorem is satisfied. The enhanced symplectic synchronization of the Chen system with uncertain parameters and the Lorenz system is achieved. The numerical results are shown in Figures 7 and 8. After 1 second, the motion trajectories enter a chaotic attractor.

\section{Conclusions}

We achieve the novel enhanced symplectic synchronization of a Chen system with uncertain parameters, and a Lorenz system is obtained by the Lyapunov asymptotical stability 

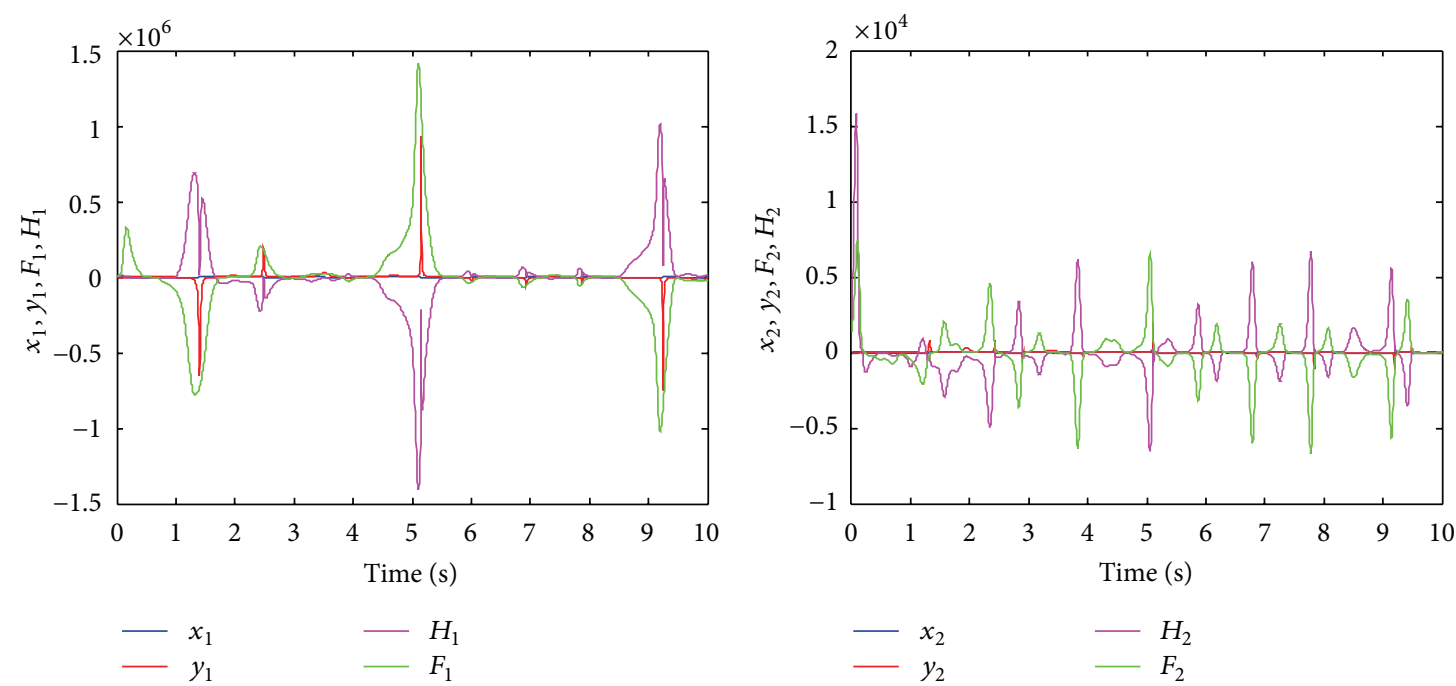

(a)
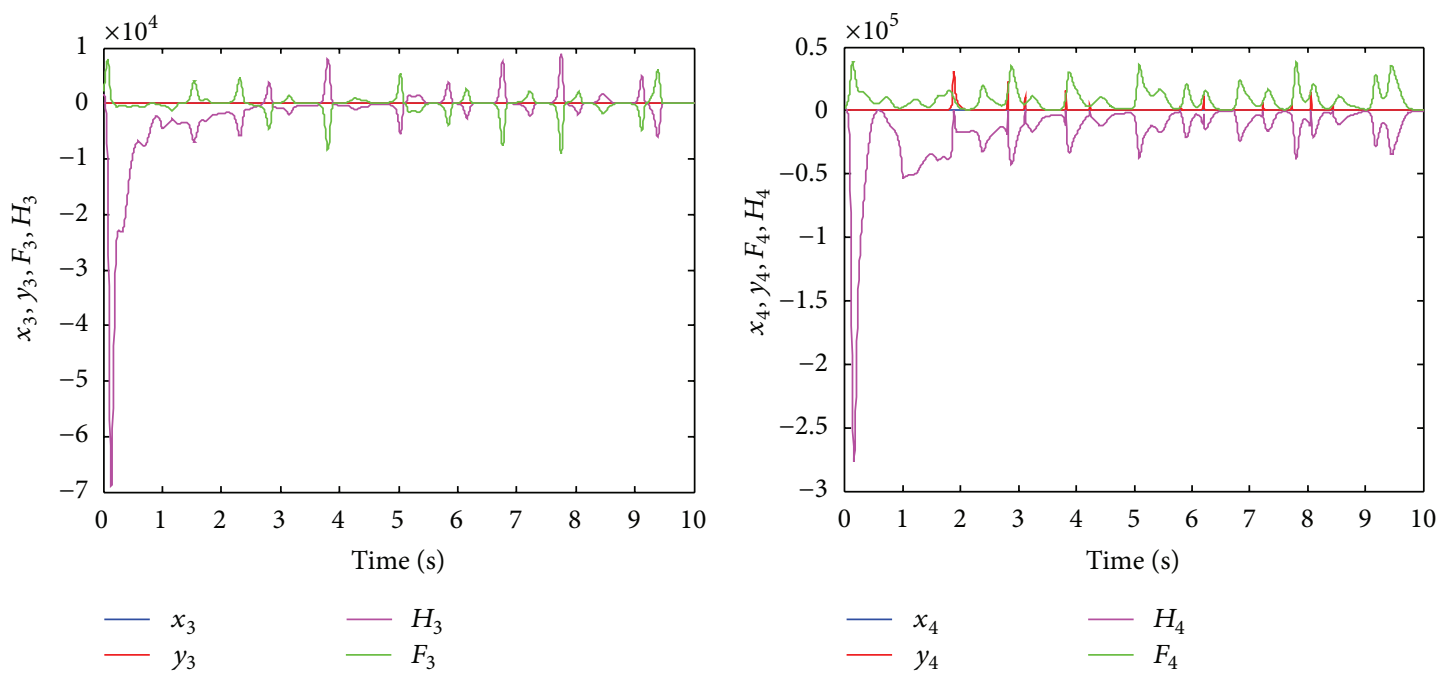

(c)

(d)

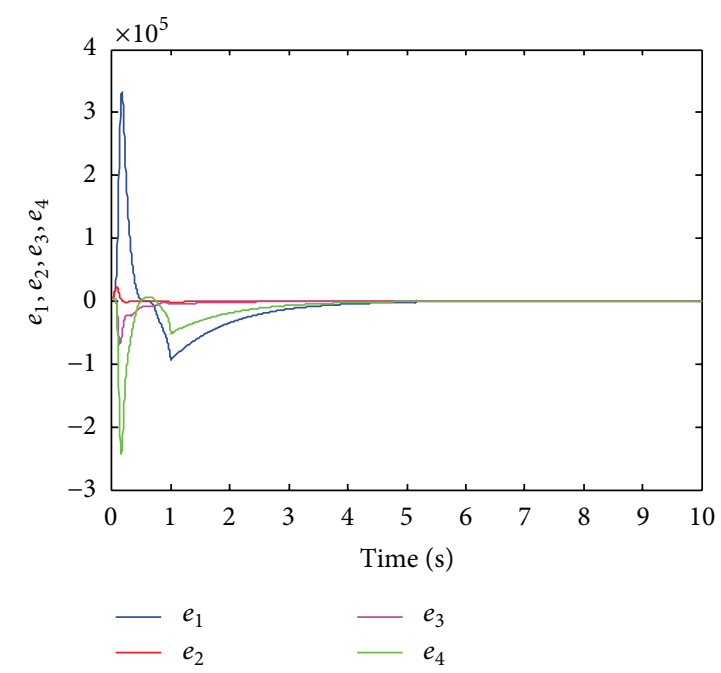

(e)

FIGURE 8: Time histories of states, state errors, $F_{1}, F_{2}, F_{3}, F_{4}, H_{1}, H_{2}, H_{3}$, and $H_{4}$ for Case 3. 
theorem. All the theoretical results are verified by numerical simulations to demonstrate the effectiveness of the three cases of proposed synchronization schemes. The enhanced symplectic synchronization of chaotic systems with uncertain parameters can be used to increase the security of secret communication.

\section{Acknowledgment}

This research was supported by the National Science Council, Taiwan, under Grant no. 98-2218-E-011-010.

\section{References}

[1] Z. M. Ge and C. H. Yang, "The generalized synchronization of a Quantum-CNN chaotic oscillator with different order systems," Chaos, Solitons and Fractals, vol. 35, no. 5, pp. 980-990, 2008.

[2] Z.-M. Ge and C.-H. Yang, "Synchronization of complex chaotic systems in series expansion form," Chaos, Solitons and Fractals, vol. 34, no. 5, pp. 1649-1658, 2007.

[3] L. M. Pecora and T. L. Carroll, "Synchronization in chaotic systems," Physical Review Letters, vol. 64, no. 8, pp. 821-824, 1990.

[4] Z. M. Ge and W. Y. Leu, "Anti-control of chaos of two-degreesof-freedom loudspeaker system and chaos synchronization of different order systems," Chaos, Solitons and Fractals, vol. 20, no. 3, pp. 503-521, 2004.

[5] R. Femat, J. Alvarez-Ramírez, and G. Fernández-Anaya, “Adaptive synchronization of high-order chaotic systems: a feedback with lower-order parametrization," Physica D, vol. 139, no. 3-4, pp. 231-246, 2000.

[6] Z. M. Ge and C. M. Chang, "Chaos synchronization and parameters identification of single time scale brushless DC motors," Chaos, Solitons and Fractals, vol. 20, no. 4, pp. 883-903, 2004.

[7] Z. An, H. Zhu, X. Li, C. Xu, Y. Xu, and X. Li, "Nonidentical linear pulse-coupled oscillators model with application to time synchronization in wireless sensor networks," IEEE Transactions on Industrial Electronics, vol. 58, no. 6, pp. 2205-2215, 2011.

[8] C.-H. Chen, L.-J. Sheu, H.-K. Chen et al., "A new hyper-chaotic system and its synchronization," Nonlinear Analysis. Real World Applications, vol. 10, no. 4, pp. 2088-2096, 2009.

[9] X. Guo, W. Wu, and Z. Chen, "Multiple-complex coefficientfilter-based phase-locked loop and synchronization technique for three-phase grid-interfaced converters in distributed utility networks," IEEE Transactions on Industrial Electronics, vol. 58, no. 4, pp. 1194-1204, 2011.

[10] Y. Huang, Y. W. Wang, and J. W. Xiao, "Generalized lagsynchronization of continuous chaotic system," Chaos, Solitons and Fractals, vol. 40, no. 2, pp. 766-770, 2009.

[11] Z. M. Ge and C. H. Yang, "Symplectic synchronization of different chaotic systems," Chaos, Solitons and Fractals, vol. 40, no. 5, pp. 2532-2543, 2009.

[12] Z. Yan, "Controlling hyperchaos in the new hyperchaotic Chen system," Applied Mathematics and Computation, vol. 168, no. 2, pp. 1239-1250, 2005.

[13] Q. Jia, "Projective synchronization of a new hyperchaotic Lorenz system," Physics Letters A, vol. 370, pp. 40-45, 2007.

[14] M. Vidyasagar, Nonlinear System Analysis, Prentice Hall, Upper Saddle River, NJ, USA, 2nd edition, 1993.
[15] M. Khalil, Nonlinear Systems, Prentice Hall, Upper Saddle River, NJ, USA, 2nd edition, 1996.

[16] C.-H. Yang, S.-Y. Li, and P.-C. Tsen, "Synchronization of chaotic system with uncertain variable parameters by linear coupling and pragmatical adaptive tracking," Nonlinear Dynamics, vol. 70, no. 3, pp. 2187-2202, 2012.

[17] Z.-M. Ge and Y.-S. Chen, "Synchronization of unidirectional coupled chaotic systems via partial stability," Chaos, Solitons and Fractals, vol. 21, no. 1, pp. 101-111, 2004.

[18] S. Chen and J. Lü, "Synchronization of an uncertain unified chaotic system via adaptive control," Chaos, Solitons and Fractals, vol. 14, no. 4, pp. 643-647, 2002.

[19] C.-H. Yang, T.-W. Chen, S.-Y. Li, C.-M. Chang, and Z.-M. Ge, "Chaos generalized synchronization of an inertial tachometer with new Mathieu-Van der Pol systems as functional system by GYC partial region stability theory," Communications in Nonlinear Science and Numerical Simulation, vol. 17, no. 3, pp. 1355-1371, 2012. 


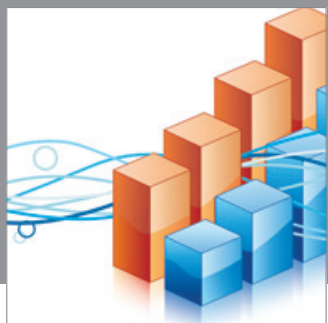

Advances in

Operations Research

mansans

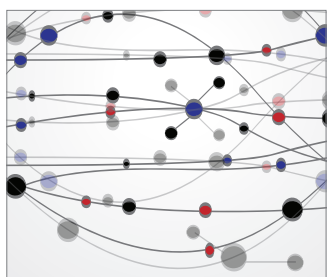

The Scientific World Journal
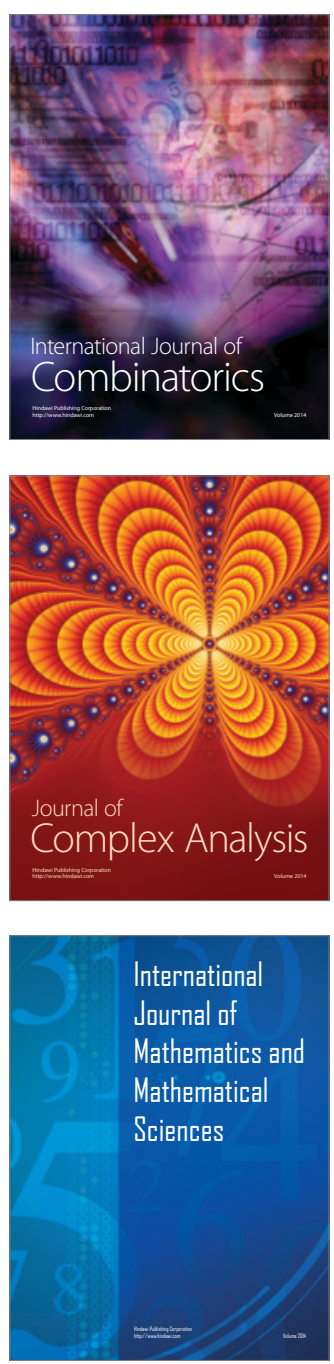
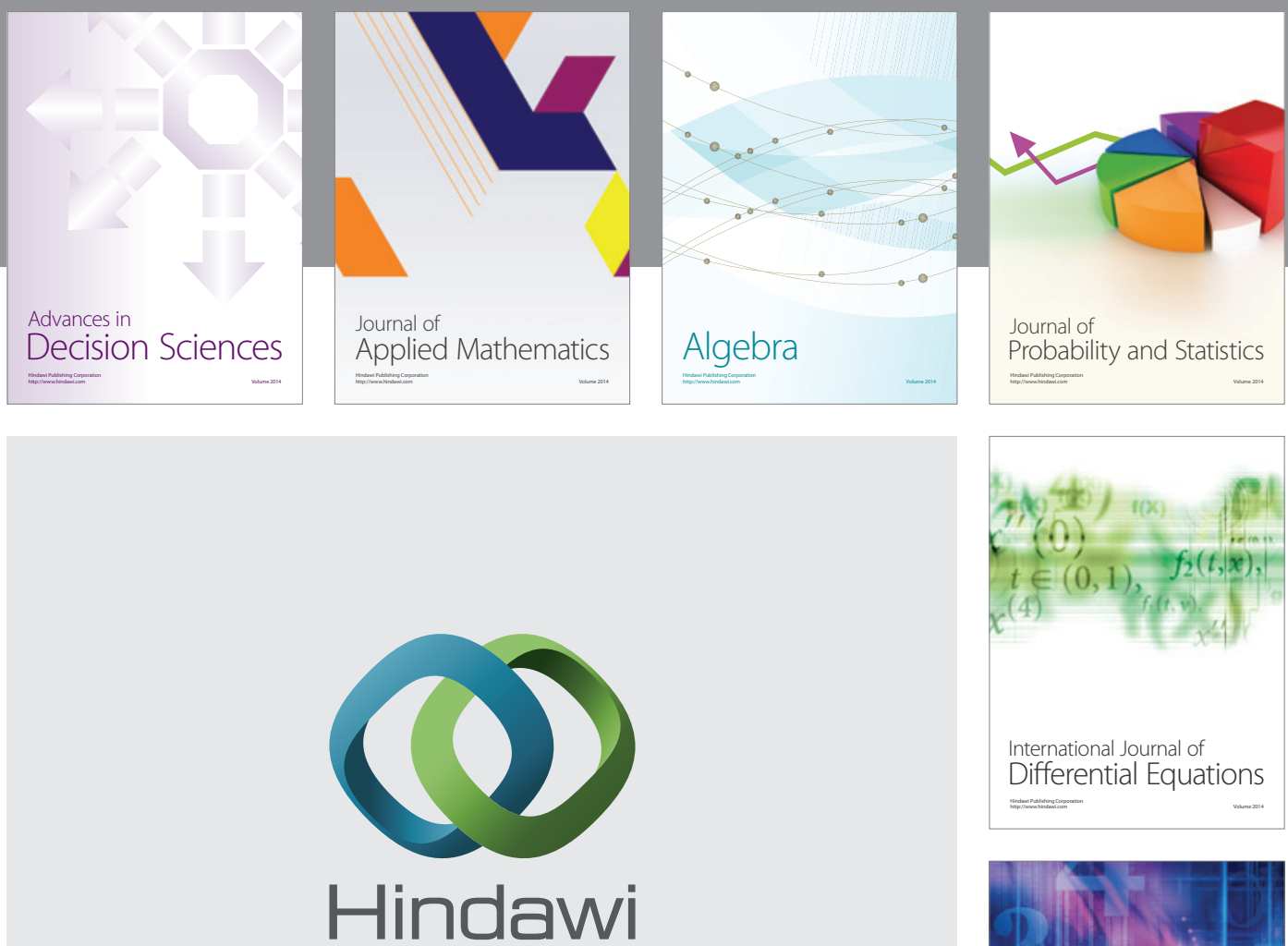

Submit your manuscripts at http://www.hindawi.com
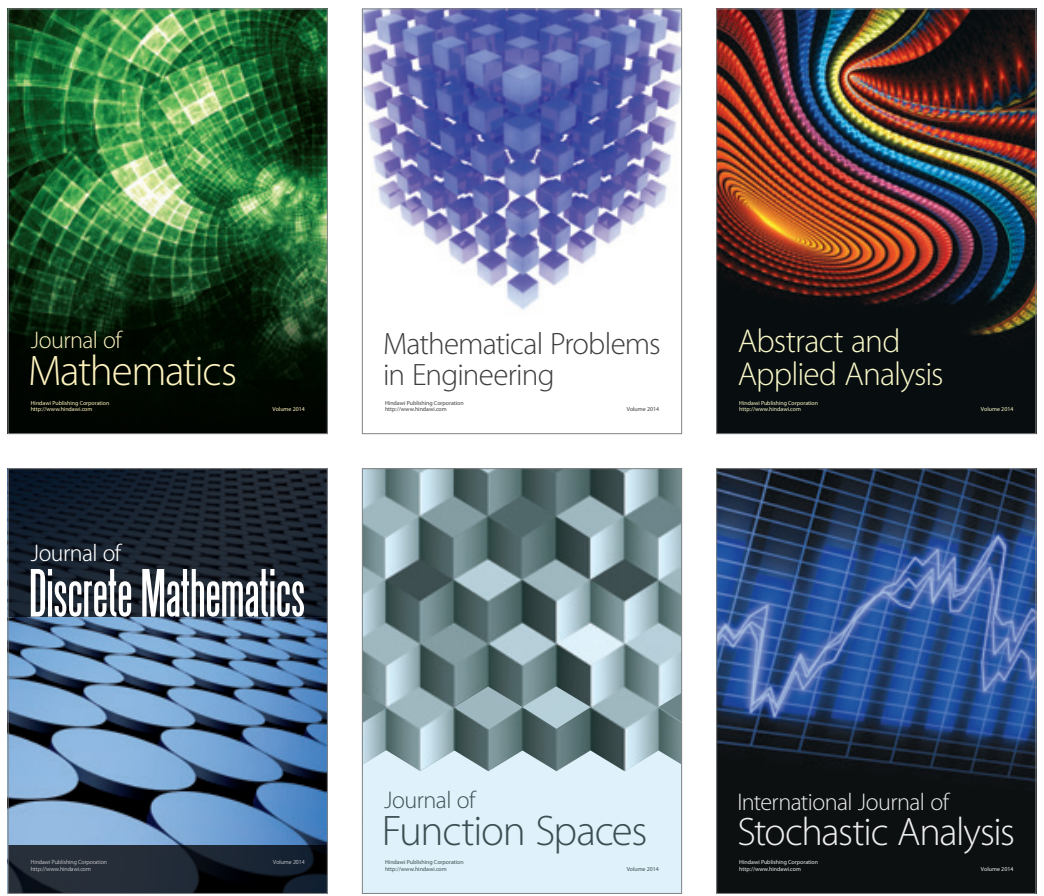

Journal of

Function Spaces

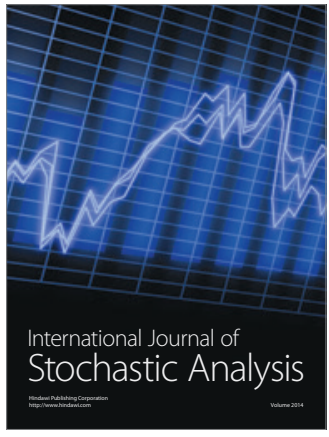

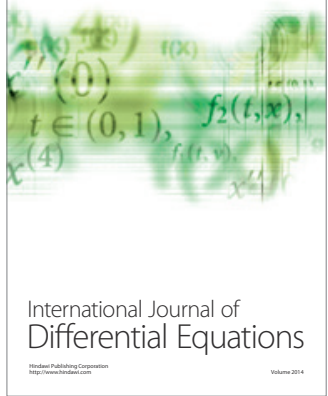
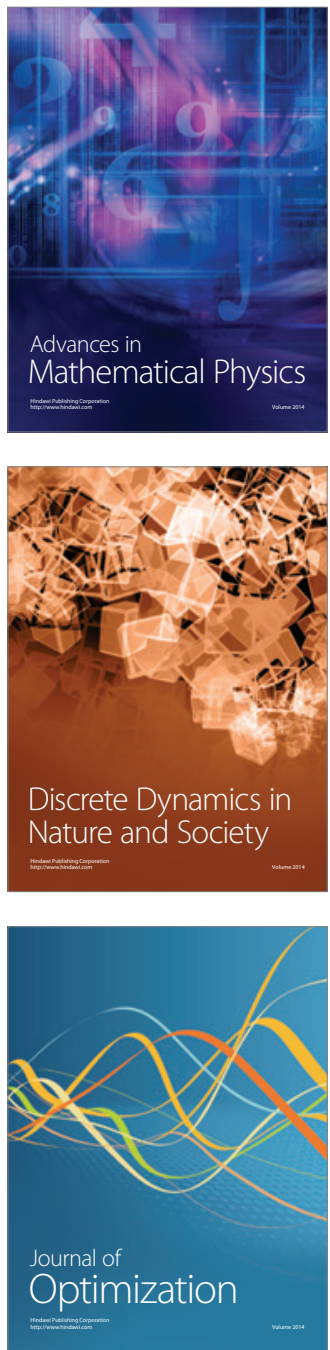